\title{
High-Gain High-Efficiency IPOS LLC Converter With Coupled Transformer and Current Sharing Capability
}

\author{
Yucen Li, Shuai Shao, Hui Chen, Junming Zhang, and Kuang Sheng
}

\begin{abstract}
In this paper, an input-parallel output-series (IPOS) LLC resonant converter with a coupled transformer and current sharing capability is proposed for high-gain high-efficiency applications such as the renewable energy grid connection. The coupled transformer is utilized to reduce the core size and core loss by implementing the magnetic flux cancellation method, which achieves a natural balance of output voltage simultaneously. The current sharing between the converter modules is achieved by a bridge wire that interconnects the resonant tanks of different modules. Furthermore, the current sharing inside a module is also achieved by the proposed compensation current based on the coordination of compensation inductor and delay control. A $200 \mathrm{kHz} 100 \mathrm{~V} / 1.2 \mathrm{kV} 3.6 \mathrm{~kW}$ two-module IPOS LLC resonant converter prototype is built to verify the proposed method. The peak efficiency can reach as high as $\mathbf{9 7 . 5 \%}$, and the current sharing between modules and inside a module are well presented.
\end{abstract}

Index Terms - Current sharing, coupled transformer, LLC converter, input-parallel output-series (IPOS).

\section{INTRODUCTION}

$\mathrm{R}$ ENEWABLES accounted for almost half of the growth in power generation (49\%) in 2017, and led by wind and solar, the share of renewables in global power generation increased from $7.4 \%$ to $8.4 \%$ [1], [2]. With the increasing penetration renewables, dc distribution systems are becoming the preferred choice in the utility grid as they provide more natural interface with renewables including solar, fuel cells and wind turbine based on permanent-magnet generators, as well as energy storage including battery and super capacitors. In addition, de distribution system has better current carrying capacity and faster dynamic response when compared to conventional ac systems.

To interconnect the renewables such as solar panel to the

Manuscript received August 23, 2019; revised November 20, 2019; accepted December 27, 2019. Date of publication March 31, 2020; date of current version February 20, 2020. This work was supported in part by the National Key Research and Development Program of China under Grant 2018YFB0904100 and in part by the Science and Technology Project of State Grid under Grant SGHB0000KXJS1800685.

Y. Li, S. Shao, J. Zhang and K. Sheng are with the College of Electrical Engineering, Zhejiang University, Hangzhou 310027, China (e-mail: yucenlee@zju.edu.cn; shaos@zju.edu.cn; zhangjm@zju.edu.cn; shengk@zju. edu.cn).

H. Chen is with the School of Information and Electrical Engineering, Zhejiang University City College, Hangzhou 310015, China (e-mail: chenh@ zucc.edu.cn).

Digital Object Identifier 10.24295/CPSSTPEA.2020.00006

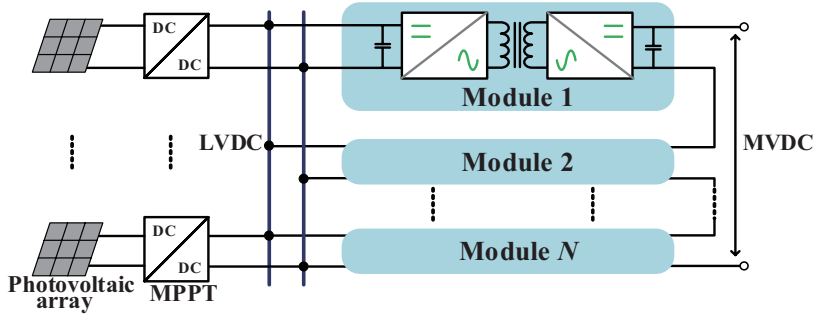

Fig. 1. IPOS configuration for renewables grid connection.

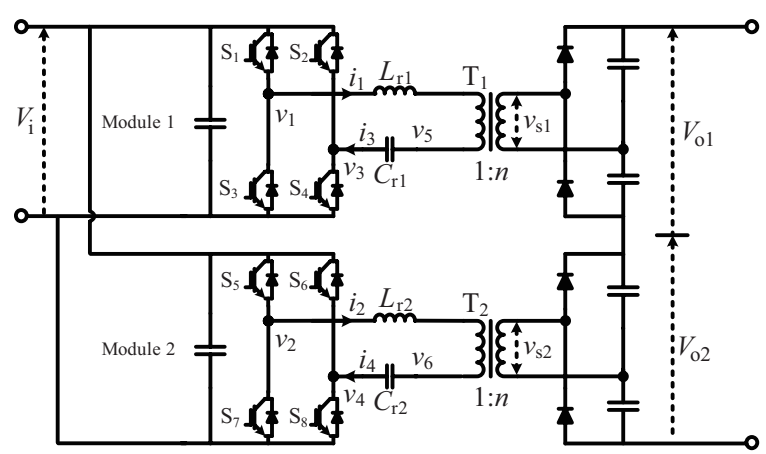

Fig. 2. IPOS LLC resonant converter.

medium voltage grid, high-gain DC-DC converters are usually required. The input-parallel output-series (IPOS) configuration (Fig. 1) is very attractive in high input current and high output voltage applications [3]-[7]. Due to the parallel and series connection of inputs and outputs, the current and voltage stress on each module is reduced, which allows the use of lowvoltage power devices with low on-resistance and enables a higher system efficiency.

In the grid connected applications, the voltage is usually maintained stable for both the low-voltage (LV) and mediumvoltage (MV) side. Therefore, an unregulated DC-DC transformer (DCX) can be used to serve as the DC-DC module in IPOS circuit, which helps to improve the efficiency and simplify the control of the isolated stage. The full-bridge converters with phase-shift control have been widely employed in high-power conversion [8]-[12], which is however hard to achieve zero-voltage-switching (ZVS) at light load condition and usually has a high turn-off current [13], [14]. Thus, the efficiency is hard to further improve.

As shown in Fig. 2, the LLC resonant converter is becoming one of the most attractive choices because it can achieve ZVS for primary switches and zero-current switching (ZCS) 
for secondary rectifiers simultaneously [15], [16], resulting in a high efficiency and low electromagnetic interference. The excellent soft switching characteristics also enable the LLC resonant converter operating at a higher frequency and achieving a higher power density. Besides, the LLC converter is particularly suitable for DCX applications because it can run at the fixed resonant frequency and therefore the efficiency benefits can be maximized [16].

For the IPOS configuration, balancing of input currents and output voltages is vital for a reliable and high efficient operation. The relationship between output voltage and input current sharing was revealed in [17] and an output voltage sharing loop was proposed. A current sharing method for interleaved LLC converter with partial energy processing is presented in [18], where a transformer and auxiliary dc-dc converters are added for current sharing loops. However, the additional voltage current sharing loop requires a complex control strategy, which becomes an obstacle when the LLC converter operates as a DCX.

To achieve the automatic current sharing, a common passive impedance method was proposed in [19]-[21], which exhibits a good performance in multi-phase half-bridge LLC converters. However, the $\Delta / Y$ equivalent circuit method proposed in [19] and [20] is not applicable to full-bridge LLC converters when current sharing both between modules and inside a module needs consideration and therefore, a different manifest model is required. Besides, the current flows through the interconnection inductor is not under precise control for the passive compensation.

This paper proposes a high-gain high-efficiency IPOS LLC converter with coupled transformer and current sharing capability. To improve efficiency, the magnetic flux cancellation concept is utilized to construct a coupled transformer, which can not only reduce the core size and the core loss, but also balances the output voltage between modules as well. To achieve resonant current sharing between modules, a structure that utilizes a bridge wire to interconnect the resonant tanks between modules is proposed and the equivalent model is given to present the cause and solution for the current unbalance. In order to further achieve the current sharing inside a module, an active control method by coordinating the compensation inductor and delay control is proposed to generate compensation current. At last, to verify the proposed IPOS LLC converter, a laboratory prototype was built with rated parameters of 200 $\mathrm{kHz} 100 \mathrm{~V} / 1.2 \mathrm{kV} 3.6 \mathrm{~kW}$. The experimental results show the proposed converter has a good performance on current sharing and a peak efficiency of $97.5 \%$.

\section{Coupled Transformer With Flux Cancellation}

An IPOS LLC resonant converters contains multiple DCDC modules, and each module requires a transformer, and these transformers can account for a large part of the whole converter loss, thus the transformer design is critical. Magnetic flux cancellation can reduce the core losses and size, and a matrix transformer configuration with magnetic cancellation is proposed in [22], and this concept is applied to the IPOS LLC

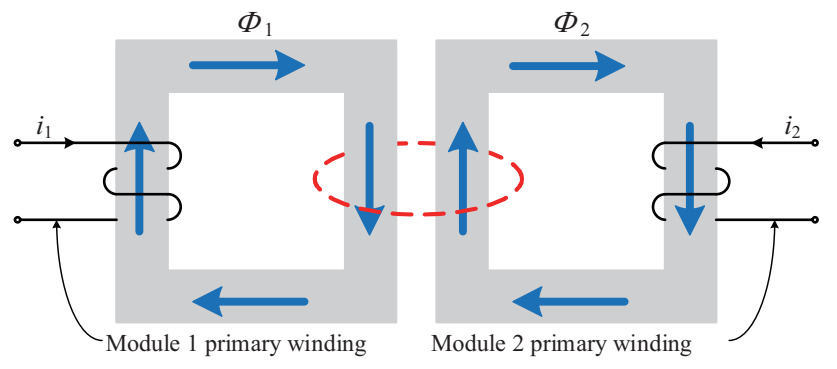

(a)

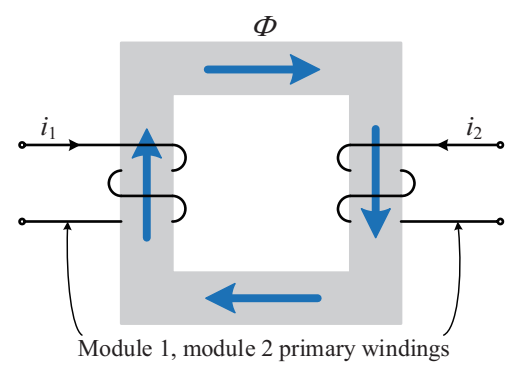

(b)

Fig. 3. Diagram of transformer. (a) With separate cores. (b) Coupled by a common core.

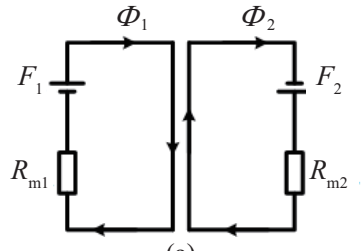

(a)



(b)
Fig. 4. Equivalent magnetic circuit of transformer.

converter to reduce the core losses.

The concept of the magnetic flux cancellation is shown in Fig. 3. When two separate magnetic cores are adopted (Fig. 3(a)), properly arranging primary windings of the two modules can let $\Phi_{1}$ and $\Phi_{2}$, the fluxes in core 1 and core 2, flow in the same direction. When the winding current $i_{1}$ and $i_{2}$ of two modules are the same, the fluxes in the neighbor legs of core 1 and core 2 are of the same magnitude but in reverse direction. Fig. 4 shows the equivalent magnetic circuit model of Fig. 3, and we can derive:

$$
\Phi_{1}=\Phi_{2}=\Phi
$$

when

$$
\left\{\begin{array}{l}
F_{1}=F_{2} \\
R_{\mathrm{m} 1}=R_{\mathrm{m} 2}
\end{array} .\right.
$$

Therefore, the center cylinder of two cores can be reaped and the two transformers can be implemented using a single U-shape core, as shown in Fig. 3(b). Despite the elimination of center cylinder, the magnetic flux flows in the remaining part of core is unchanged. Hence, the core size and the core loss are 


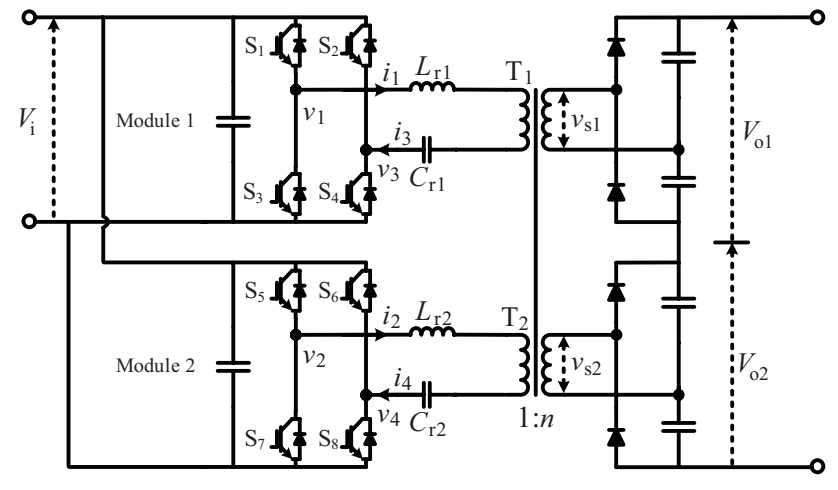

Fig. 5. IPOS LLC converter with coupled transformer.

well reduced.

With the coupled transformer in Fig. 3(b), the magnetic flux flows through winding of two modules is forced to be identical. The voltage on the secondary side of transformer can be derived as follows:

$$
v_{\mathrm{s} 1}=v_{\mathrm{s} 2}=n \frac{\mathrm{d} \Phi}{\mathrm{d} t} .
$$

According to (1), the secondary side voltage of two modules $v_{\mathrm{s} 1}$ and $v_{\mathrm{s} 2}$ can be naturally balanced, therefore output voltage $V_{\mathrm{o} 1}$ and $V_{\mathrm{o} 2}$ in Fig. 2 are naturally balanced.

\section{Current Sharing Between Modules}

For a converter with multiple modules, it is essential to distribute current evenly among different modules to allow the thermal balance and to ensure stable operation. When the transformers of the IPOS modules are coupled, as shown in Fig. 5, current sharing between modules becomes critical. To simplify the analysis, gate signals of the power devices of two modules in Fig. 5 are considered the same and without delays, we obtain:

$$
\left\{\begin{array}{l}
v_{1}=v_{2} \\
v_{3}=v_{4}
\end{array}\right.
$$

When the two modules operate nears the resonant frequency, based on fundamental harmonic approximation (FHA) [23], [24], an equivalent circuit is shown in Fig. 6. The voltage relationship between input and output is expressed as follows:

where

$$
\left\{\begin{array}{l}
v_{13}=i_{1} \cdot Z_{1}+\frac{v_{\mathrm{s} 1}}{n} \\
v_{24}=i_{2} \cdot Z_{2}+\frac{v_{\mathrm{s} 2}}{n}
\end{array},\right.
$$

$$
\left\{\begin{array}{l}
Z_{1}=j \omega L_{\mathrm{r} 1}+\frac{1}{j \omega C_{\mathrm{r} 1}} \\
Z_{2}=j \omega L_{\mathrm{r} 2}+\frac{1}{j \omega C_{\mathrm{r} 2}}
\end{array}\right.
$$

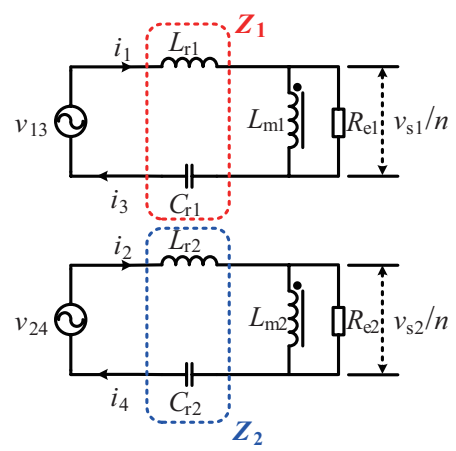

Fig. 6. Equivalent circuit of IPOS LLC converter.

Based on (1), (2) and (3), the relative relationship between the resonant currents of the two modules can be derived:

$$
\frac{i_{1}}{i_{2}}=\frac{Z_{2}}{Z_{1}}=\frac{j \omega L_{\mathrm{r} 2}+\frac{1}{j \omega C_{\mathrm{r} 2}}}{j \omega L_{\mathrm{r} 1}+\frac{1}{j \omega C_{\mathrm{r} 1}}}
$$

Current unbalance between modules is mainly attributed to the component tolerance in the resonant inductor or capacitor. Considering 5\% tolerance in $L_{\mathrm{r} 1}$ and $C_{\mathrm{r} 1}$ respectively, and we use $\left|\left(i_{1}-i_{2}\right) / i_{2}\right|$ to evaluate the relative error of currents between two modules. Fig. 7 shows the relative error of the currents changing with the resonant components $L_{\mathrm{r} 2}$ and $C_{\mathrm{r} 2}$ respectively, where $L_{\mathrm{r}}$ and $C_{\mathrm{r}}$ represent the nominal resonant inductance and capacitance. The current-sharing error increase dramatically with light different between resonant components and can reach $100 \%$ when $L_{\mathrm{r} 1}=1.05 L_{\mathrm{r} 2}$ or $C_{\mathrm{r} 1}=1.05 C_{\mathrm{r} 2}$.

To balance the current between modules, a wire bridge is inserted to short-circuit the resonant capacitors of two modules, as shown in Fig. 8, whose equivalent circuit model is shown in Fig. 9. With this short wire, we obtain:

$$
v_{5}=v_{6}
$$

Furthermore, the voltage constraints can be described as:

$$
\begin{gathered}
\left\{\begin{array}{l}
v_{15}=i_{1} \cdot j \omega L_{\mathrm{r} 1}+\frac{v_{\mathrm{s} 1}}{n} \\
v_{26}=i_{2} \cdot j \omega L_{\mathrm{r} 2}+\frac{v_{\mathrm{s} 2}}{n}
\end{array}\right. \\
\left\{\begin{array}{l}
v_{53}=i_{3} \cdot \frac{1}{j \omega C_{\mathrm{r} 1}} \\
v_{64}=i_{4} \cdot \frac{1}{j \omega C_{\mathrm{r} 2}}
\end{array}\right.
\end{gathered}
$$

Combining (3), (4), (8), (9) and (10) the relationship between the resonant currents of the two modules can be derived: 




(a)

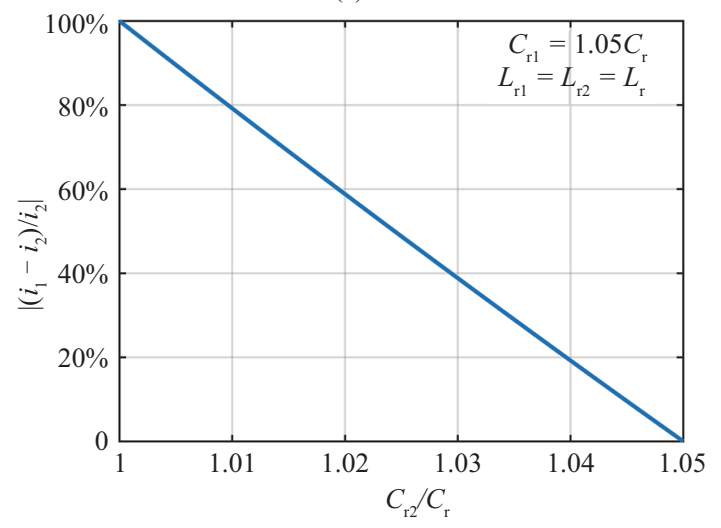

(b)

Fig. 7. Current sharing error versus. (a) Inductance tolerance. (b) Capacitance tolerance.

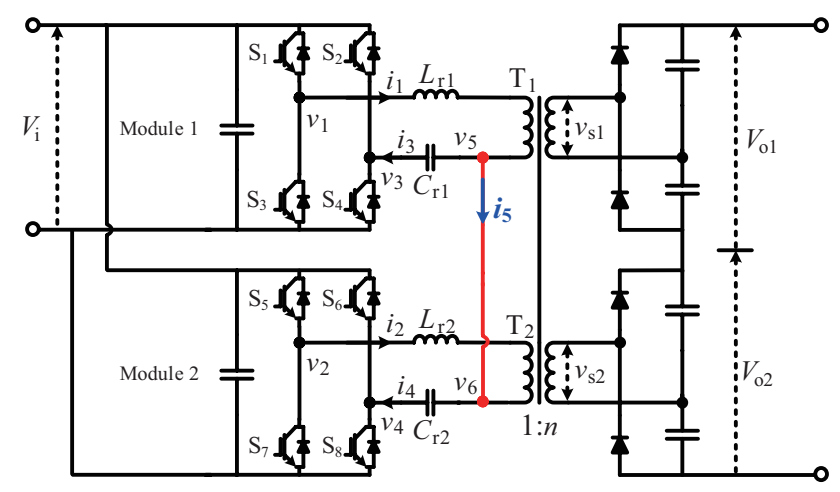

Fig. 8. IPOS LLC converter with short-circuit resonant capacitors.

$$
\left\{\begin{array}{l}
\frac{i_{1}}{i_{2}}=\frac{L_{\mathrm{r} 2}}{L_{\mathrm{r} 1}} \\
\frac{i_{3}}{i_{4}}=\frac{C_{\mathrm{r} 1}}{C_{\mathrm{r} 2}}
\end{array} .\right.
$$

Still consider 5\% tolerance in $L_{\mathrm{r} 1}$ and $C_{\mathrm{r} 1}$ respectively, and Fig. 10 shows the current sharing error IPOS modules using the proposed balancing technique. The current-sharing is considerably improved, and even in the worst case, no more than $5 \%$ error is observed, which is acceptable in the practice

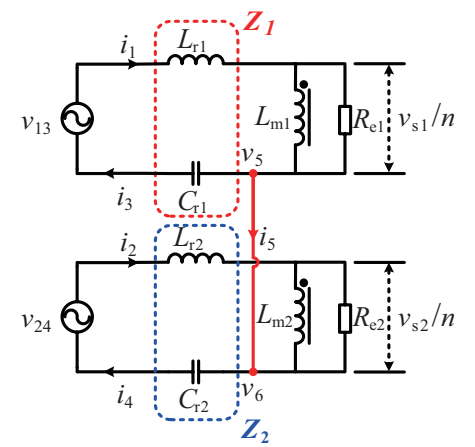

Fig. 9. Equivalent circuit with short-circuit resonant capacitors.

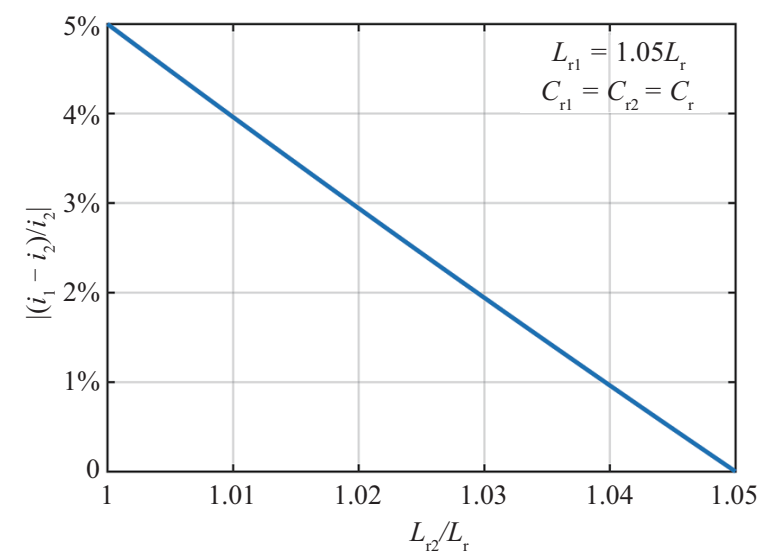

(a)

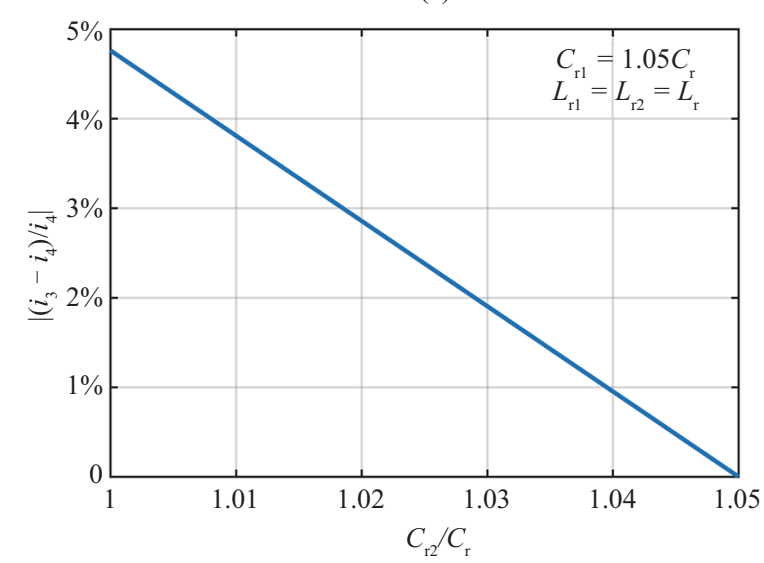

(b)

Fig. 10. Current sharing error versus. (a) Inductance tolerance. (b) Capacitance tolerance.

since the primary purpose of current sharing is to achieve thermal balance. The current sharing can be further improved by choosing resonant components with smaller tolerance.

\section{Current Sharing Inside a Module}

The interconnection of resonant tanks can balance the current between modules $\left(i_{1}\right.$ and $i_{2}, i_{3}$ and $\left.i_{4}\right)$, but current inside a module can still be different $\left(i_{1}\right.$ and $i_{3}, i_{2}$ and $i_{4}$ ) because the current $i_{5}$ in Fig. 9 that flows through the interconnection wire: 


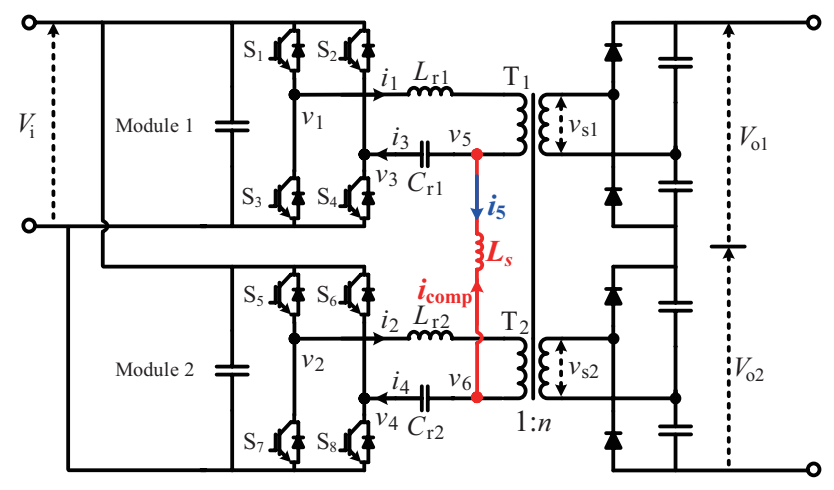

Fig. 11. IPOS LLC converter with compensation inductor.

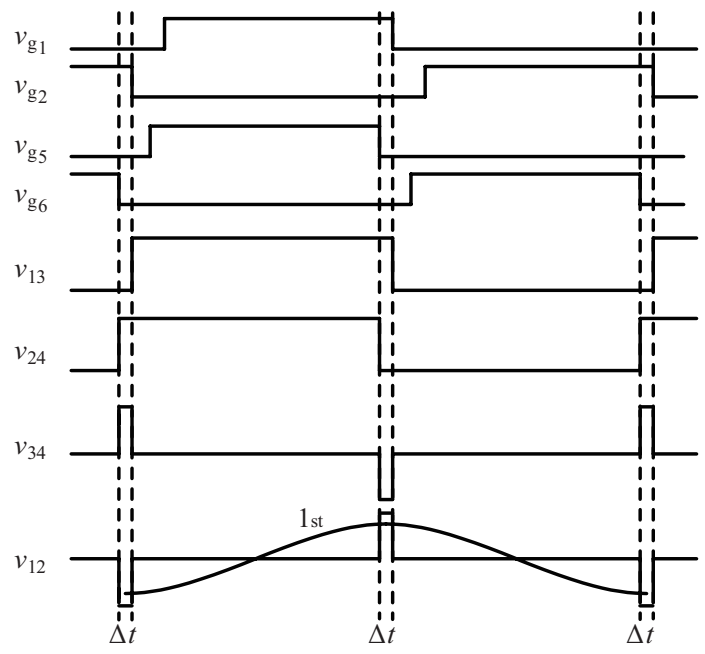

(a)



(b)

Fig. 12. (a) Gate signals and H-bridge output voltages. (b) Resonant currents.

$$
i_{5}=i_{1}-i_{3}=i_{4}-i_{2}
$$

The current $i_{5}$ reflects the difference between $i_{1}$ and $i_{3}, i_{2}$ and $i_{4}$. To balance the current inside a module, additional method is proposed to suppress the current $i_{5}$. A compensation inductor $L_{\mathrm{s}}$ is utilized to replace the interconnection wire, as shown in Fig. 11. With proper gate signal delay control, a compensation current $i_{\text {comp }}$ can be generated to offset $i_{5}$ and balance the currents inside a module.

To analyze the principle for generating compensation current $i_{\text {comp }}$, consider the gate signals of module 1 lag behind that of module 2 by a tiny period of time $\Delta t$, as shown in Fig. 12(a).

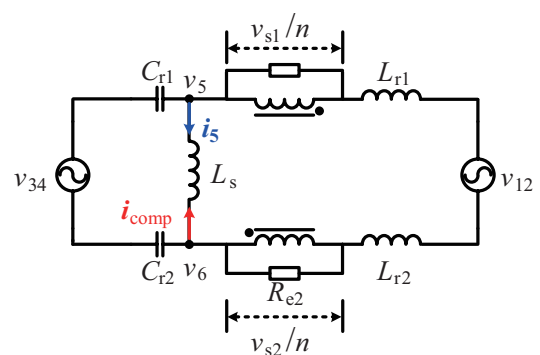

Fig. 13. Equivalent circuit under narrow pulse voltages.



Fig. 14. Simplified equivalent circuit under narrow pulse voltages.

As a result, the square voltage $v_{13}$ (module 1 ) will fall behind $v_{24}$ (module 2) by $\Delta t$, which will produce narrow pulse voltages $v_{12}$ and $v_{34}$. From the waveform, the phase of $v_{12}$ is $90^{\circ}$ behind the square voltage $v_{13}$ and $v_{24}$ generated by H-bridges. Besides, $v_{12}$ and $v_{34}$ are of the same magnitude but in reverse phase:

$$
v_{12}=-v_{34}
$$

The pulse $v_{12}$ and $v_{34}$ can be employed to balance the current inside a module. Fig. 13 shows the equivalent circuit. Because the coupled transformer shares the same core and $v_{\mathrm{s} 1}=v_{\mathrm{s} 2}$, as described in (1), the equivalent circuit can be further simplified as Fig. 14. Ignore the tolerance of resonant components, we obtain:

$$
\left\{\begin{array}{l}
L_{\mathrm{r} 1}=L_{\mathrm{r} 2}=L_{\mathrm{r}} \\
C_{\mathrm{r} 1}=C_{\mathrm{r} 2}=C_{\mathrm{r}} \\
\omega_{\mathrm{r}}=\frac{1}{\sqrt{L_{\mathrm{r}} C_{\mathrm{r}}}} .
\end{array}\right.
$$

Based on Fig. 14, the transfer function from $v_{12}$ to $i_{\text {comp }}$ can be derived, by combining with (11) and (12):

$$
\frac{i_{\text {comp }}(\omega)}{v_{12}(\omega)}=H\left(\frac{\omega}{\omega_{\mathrm{r}}}, \frac{L_{\mathrm{s}}}{L_{\mathrm{r}}}\right) \cdot \frac{1}{\omega_{\mathrm{r}} L_{\mathrm{r}}}
$$

where:

$$
H\left(\frac{\omega}{\omega_{\mathrm{r}}}, \frac{L_{\mathrm{s}}}{L_{\mathrm{r}}}\right)=\frac{\left(\frac{\omega}{\omega_{\mathrm{r}}}\right)^{2}+1}{j\left(\frac{\omega}{\omega_{\mathrm{r}}}\right)\left[\frac{L_{\mathrm{s}}}{L_{\mathrm{r}}}-\frac{L_{\mathrm{s}}}{L_{\mathrm{r}}}\left(\frac{\omega}{\omega_{\mathrm{r}}}\right)^{2}+2\right]}
$$

To analyze the characteristics of $i_{\text {comp }}$, the bode diagram 


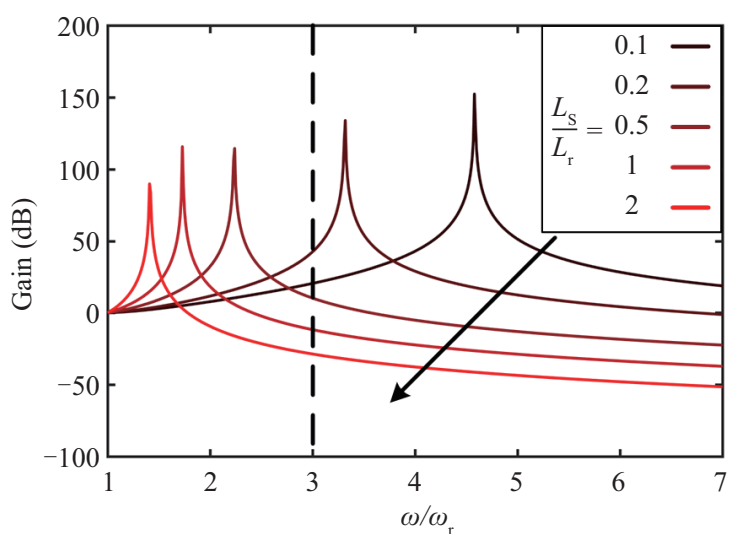

(a)

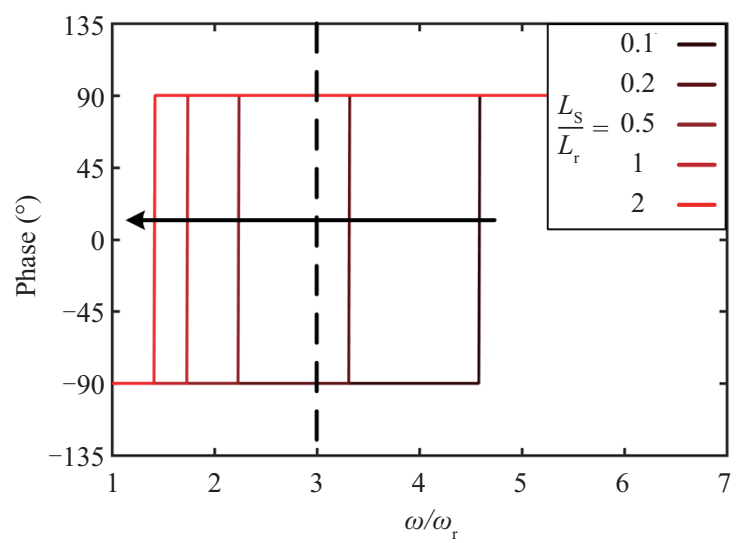

(b)

Fig. 15. The bode diagram of H. (a) Amplitude-frequency. (b) Phase-frequency.

of $H\left(\omega / \omega_{\mathrm{r}}, L_{\mathrm{s}} / L_{\mathrm{r}}\right)$ is depicted in Fig. 15, where different compensation inductance $L_{\mathrm{s}}$ are considered. As shown in Fig. 15 (b), the fundamental harmonic component of $i_{\text {comp }}$ always lags behind $v_{12}$ by $90^{\circ}$, while $v_{12}$ lags behind the unbalanced current $i_{5}$ by $90^{\circ}$, as illustrated in Fig. 12. As a result, the fundamental harmonic component of $i_{\text {comp }}$ lags behind $i_{5}$ by $180^{\circ}$, which can be utilized to offset the unbalanced current $i_{5}$.

It is worth noting that in practice, the direction of current $i_{5}$ is determined by the relative magnitude of the resonant component values. Based on Fig. 9, (11) and (12), we can derive:

$$
i_{5}=\frac{\frac{C_{\mathrm{r} 2}}{C_{\mathrm{r} 1}}-\frac{L_{\mathrm{r} 1}}{L_{\mathrm{r} 2}}}{1+\frac{C_{\mathrm{r} 2}}{C_{\mathrm{r} 1}}} \cdot i_{1} .
$$

When $C_{\mathrm{r} 2} / C_{\mathrm{r} 1}>L_{\mathrm{r} 1} / L_{\mathrm{r} 2}$, the current $i_{5}$ flows in the same phase to $i_{1}$, as depicted in 13(b), which requires the gate signals of module 1 lagging behind that of module 2 as discussed above. When $C_{\mathrm{r} 2} / C_{\mathrm{r} 1}<L_{\mathrm{r} 1} / L_{\mathrm{r} 2}$, the current $i_{5}$ flows in the opposite phase to $i_{1}$. Therefore, the gate signals of module 1 should be adjusted to lead ahead of that of module 2 to generate the compensation current in opposite phase.
Meanwhile, the higher order harmonics of $i_{\text {comp }}$ needs to be suppressed. As shown in Fig. 15, if $L_{\mathrm{s}}$ is too small, higher order harmonics $i_{\text {comp }}$ of may be larger than its fundamental component, leading to the distortion of resonant current. Since $i_{\text {comp }}$ only contains odd harmonics, the gain of third and higher harmonics should be restricted to be smaller than fundamental harmonic, which can be expressed as:

$$
\left\{\begin{array}{l}
\left|H\left(3, \frac{L_{\mathrm{s}}}{L_{\mathrm{r}}}\right)\right| \leqslant\left|H\left(1, \frac{L_{\mathrm{s}}}{L_{\mathrm{r}}}\right)\right| \\
\angle H\left(3, \frac{L_{\mathrm{s}}}{L_{\mathrm{r}}}\right)=90^{\circ}
\end{array}\right.
$$

By solving (18), we obtain:

$$
L_{\mathrm{s}} \geqslant \frac{2}{3} L_{\mathrm{r}}
$$

Although larger $L_{\mathrm{s}}$ value seems to have a better suppression on higher order harmonics according to and Fig. 15 and (16), too large inductance will degrade the automatic current sharing between modules as discussed in Section III, in which case $v_{5}$ and $v_{6}$ cannot be deemed as equal for the large impedance introduced by $L_{\mathrm{s}}$. Therefore, the design of $L_{\mathrm{s}}$ needs a compromise and $L_{\mathrm{s}}$ is chosen as $2 / 3 L_{\mathrm{r}}$ in this work.

In order to well cancel the current $i_{5}$, a proper delay time $\Delta t$ should be chosen. The generated compensation current $i_{\text {comp }}$ (Fig. 11) should satisfy:

$$
\left|i_{\text {comp }}\left(\omega_{\mathrm{r}}\right)\right|=\left|i_{5}\right|
$$

where $i_{\text {comp }}\left(\omega_{\mathrm{r}}\right)$ can be derived from (15), and $i_{5}$ can be represented by $\varepsilon \cdot i_{1}, \varepsilon$ is defined as the error coefficient based on (17). Therefore:

$$
\left|i_{\text {comp }}\left(\omega_{\mathrm{r}}\right)\right|=\left|\frac{v_{12}\left(\omega_{\mathrm{r}}\right)}{j \omega_{\mathrm{r}} L_{\mathrm{r}}}\right|=\left|\varepsilon \cdot i_{1}\right|,
$$

$v_{12}\left(\omega_{\mathrm{r}}\right)$ is the fundamental component of narrow pulse voltage $v_{12}$ :

$$
v_{12}\left(\omega_{\mathrm{r}}\right)=\frac{2 \Delta t}{T_{\mathrm{s}}} \frac{4}{\pi} V_{i} \cdot \sin \omega_{\mathrm{r}} t
$$

$i_{1}$ is determined by the equivalent load $R_{\mathrm{e} 1}$ (FHA), as shown in Fig. 9:

$$
i_{1}=\frac{1}{R_{\mathrm{e} 1}} \frac{4}{\pi} V_{\mathrm{i}} \cdot \sin \left(\omega_{\mathrm{r}} t+90^{\circ}\right) .
$$

Combining (21), (22) and (23), the delay time $\Delta t$ is derived as:

$$
\Delta t=\frac{\varepsilon \pi L_{\mathrm{r}}}{R_{\mathrm{e} 1}}=\frac{\varepsilon \pi^{3} L_{\mathrm{r}}}{2 R_{\mathrm{L}}} \cdot n^{2} .
$$


$R_{\mathrm{L}}$ represents the load of the converter, and $n$ represents turns ratio of the transformer, as shown in Fig. 11. The larger error coefficient $\varepsilon$ requires a longer delay time $\Delta t$. Normally, the error coefficient is within the range of $0-5 \%$.

\section{EXPERIMENTAL RESULTS}

\section{A. Prototype}

To verify the proposed coupled transformer and current sharing capability of IPOS LLC resonant converter, a $200 \mathrm{kHz}$ $3.6 \mathrm{~kW}$ converter prototype is constructed as shown in Fig. 16 , and the parameter specifications are shown in Table I. The prototype serves as a dc/dc transformer with a fixed voltage gain, and operate at a fixed switching frequency.

The primary devices are IRFP4568PBF MOSFETs from Infineon. The secondary rectifiers are IDH20G120C5 SiC Schottky diodes from Infineon. Printed circuit board (PCB) planar winding structure are adopted both for the transformer and resonant inductors. The magnetic material of core is ferrite DMR95 from DMEGC. By utilizing interleaving wingdings, the leakage inductance of the transformer is $50 \mathrm{nH}$ (from primary side) as measured, which is negligible in the experiment. Therefore, the potential soft switching failure of power devices caused by the leakage inductance is suppressed [25]. Due to the implementation of current sharing, the current that flows through the compensation inductor is small, which will not cause large power dissipation. Therefore, an SMT inductor SRP7050TA-1R8M from BOURNS is chosen as the compensation inductor. The power density of the overall prototype is around $33 \mathrm{~W} / \mathrm{in}^{3}$.

A PCB planar transformer is designed to demonstrate the coupled transformer concept. The cost of the PCB with more than 4 layers is usually high. To reduce the cost, the windings of the transformer is implemented using three identical 4-layer PCBs and each PCB has 4:4 turns, as shown in Fig. 17. The primary side of three PCBs are connected in parallel, while the secondary side are connected in series, and the resultant turns ratio is $4: 12$. For each PCB board, primary and secondary windings are perfectly interleaved layer by layer, with two coils on each layer. The magnetic motive force (MMF) between windings is minimized. The PCB winding structure is optimized based on the design method proposed in [26], [27]. Therefore, the leakage inductance as well as ac winding loss of transformer can be minimized.

Fig. 18(a) shows the 2-D finite-element simulation result of the proposed coupled transformer. The result shows that the MMF between windings is fairly small, which induces a small leakage inductance as well. Besides, Fig. 18(b) shows the simulation result of a separate transformer with the same core but a single winding. By comparison, it is obvious that the magnetic field distribution in the core is nearly identical either in the coupled transformer or separate transformer, which verifies that the coupled transformer does not change the normal magnetic flux density in the core, and the loss in two separate transformer cores can be reduced to that in a coupled transformer core.

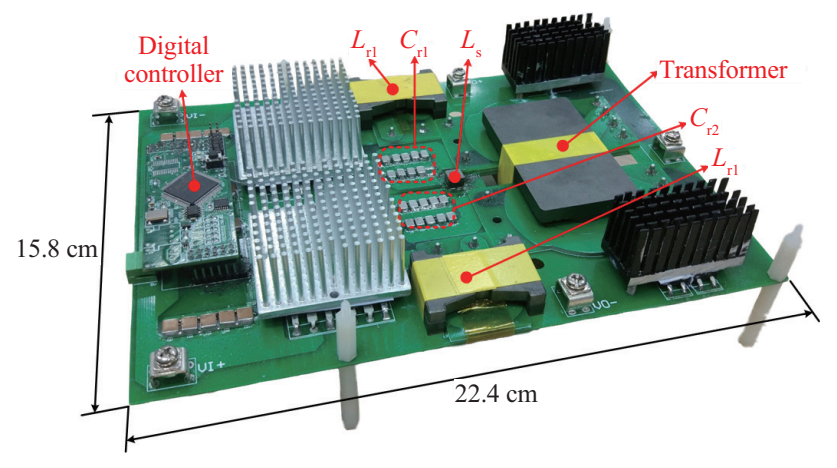

Fig. 16. Prototype of a $200 \mathrm{kHz} 3.6 \mathrm{~kW}$ converter.

TABLE I

SPECIFICATIONS OF PROTOTYPE

\begin{tabular}{lc}
\hline \hline Parameter & Value \\
\hline Switching frequency & $200 \mathrm{kHz}$ \\
Input voltage & $100 \mathrm{~V}$ \\
Output voltage & $1200 \mathrm{~V}$ \\
Dead time & $150 \mathrm{~ns}$ \\
Transformer turns ratio & $1: 3$ \\
Resonant capacitor & $216 \mathrm{nF}\left(C_{\mathrm{r} 1}\right), 238 \mathrm{nF}\left(C_{\mathrm{r} 2}\right)$ \\
Resonant inductor & $2.6 \mu \mathrm{H}\left(L_{\mathrm{r} 1}\right), 2.8 \mu \mathrm{H}\left(L_{\mathrm{r} 2}\right)$ \\
Magnetizing inductance & $25 \mu \mathrm{H}$ \\
Compensation inductor & $1.8 \mu \mathrm{H}$ \\
\hline \hline
\end{tabular}

\section{B. Experimental Waveforms}

Fig. 19 shows the experimental waveforms to validate the current sharing between modules without and with the interconnected resonant tanks respectively, which is tested under $50 \mathrm{~V}$ input, $600 \mathrm{~V}$ output and $600 \mathrm{~W}$ load condition. $v_{13}$ and $v_{24}$ are voltages on the resonant tanks of module 1 and 2 respectively. $i_{1}$ and $i_{2}$ denote the resonant currents through the resonant inductors $L_{\mathrm{r} 1}$ and $L_{\mathrm{r} 2}$ respectively. When there is no compensation inductor interconnecting two modules, approximately $30 \%$ current unbalance can be observed between two resonant tanks, as shown in Fig. 19(a). While the compensation inductor $L_{\mathrm{s}}$ is added to enable the current sharing, the two resonant currents that flows in $L_{\mathrm{r} 1}$ and $L_{\mathrm{r} 2}$ can reach nearly the same, as shown in Fig. 19(b).

Fig. 20 shows the experimental waveforms to validate the current sharing effect inside a module under different delay time between two modules, which is tested under $100 \mathrm{~V}$ input, $1.2 \mathrm{kV}$ output and $2.4 \mathrm{~kW}$ load condition. $i_{3}$ and $i_{4}$ denotes the resonant current flows through the resonant capacitors $C_{\mathrm{r} 1}$ and $C_{\mathrm{r} 2}$ respectively. No delay, $20 \mathrm{~ns}$ delay and $40 \mathrm{~ns}$ delay are tested as shown in Fig. 19. With the increase of delay time, the current difference inside a module is reduced, as shown in Fig. 20(b) and (c). Besides, the waveform of the current in interconnection inductor $L_{\mathrm{s}}$ is shown in Fig. 21. By cancelling $i_{5}$ with $i_{\text {comp }}$, the actual current flows through the inductor $L_{\mathrm{s}}$ is fairly small.

Figs. 22 and 23 show the experimental waveform under light 


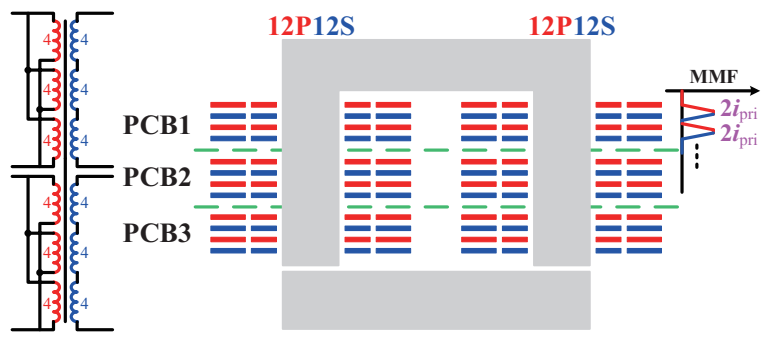

Fig. 17. Winding structure of the PCB planar transformer.

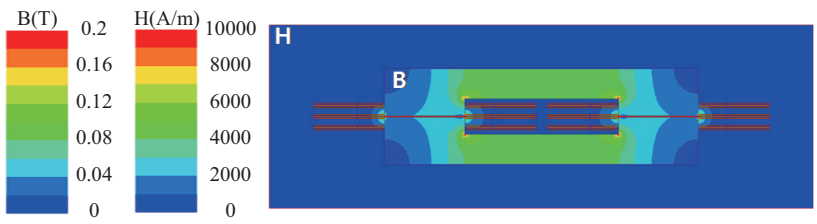

(a)

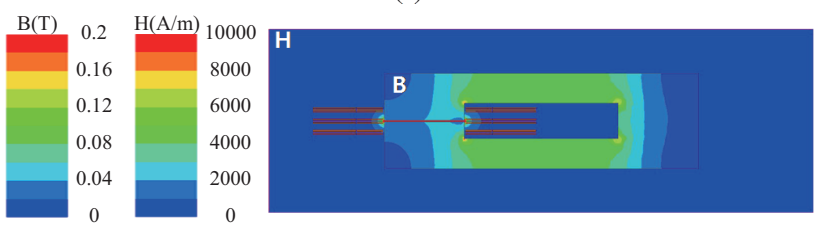

(b)

Fig. 18. 2-D finite-element simulation. (a) Coupled. (b) Separate transformer.

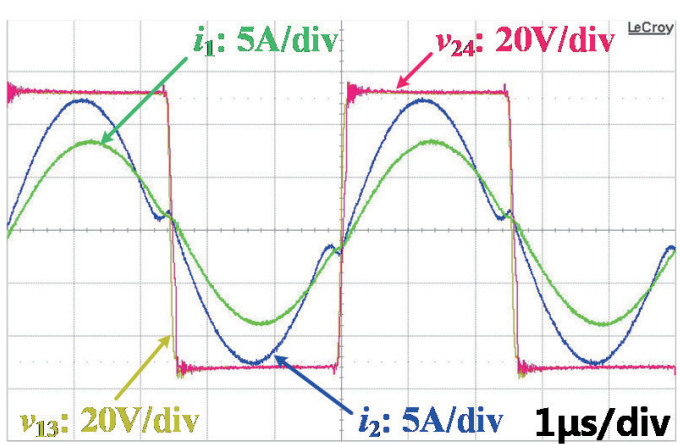

(a)

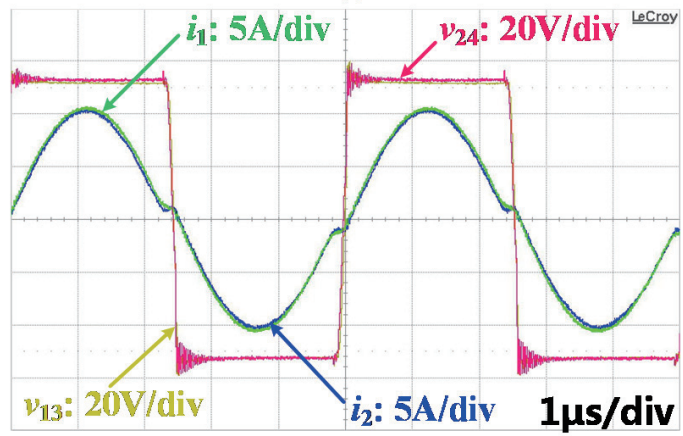

(b)

Fig. 19. Experimental waveforms for current sharing between modules. (a) Without and (b) with interconnected resonant tank.



(a)
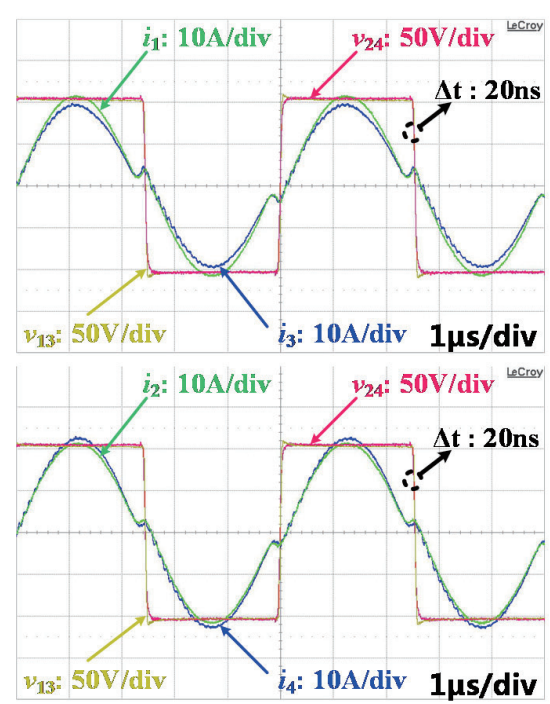

(b)
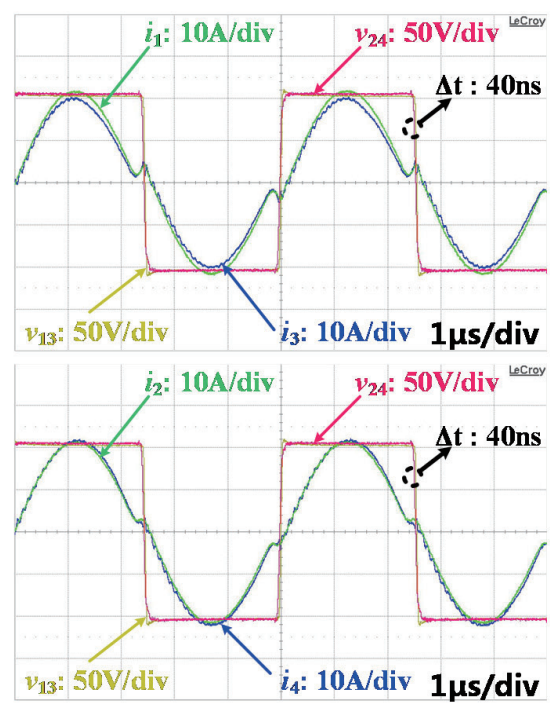

(c)

Fig. 20. Experimental waveforms for current sharing inside a module. (a) No delay time. (b) 20 ns delay. (c) 40 ns delay.

load and full load condition respectively. The oscillation under light load is attributed to the parasitic capacitor introduced by the planar transformer, which, however does not influence the normal operation of the converter and will disappear under heavy load. The results show that ZVS is achieved for primary devices of both two modules simultaneously under both light and heavy load conditions.

The measured efficiency curve of the proposed IPOS
LLC converter is shown in Fig. 24, where the efficiency of a single LLC converter is also plotted. The output power of two converters are normalized for comparison. The proposed IPOS LLC converter has a peak efficiency of $97.5 \%$ at 2.4 $\mathrm{kW}$ load and a full-load efficiency of $96.5 \%$. It is obvious that the efficiency of the proposed IPOS LLC converter is higher than that of a single LLC converter over the load range, due to the optimized coupled transformer. With rated $100 \mathrm{~V}$ input 


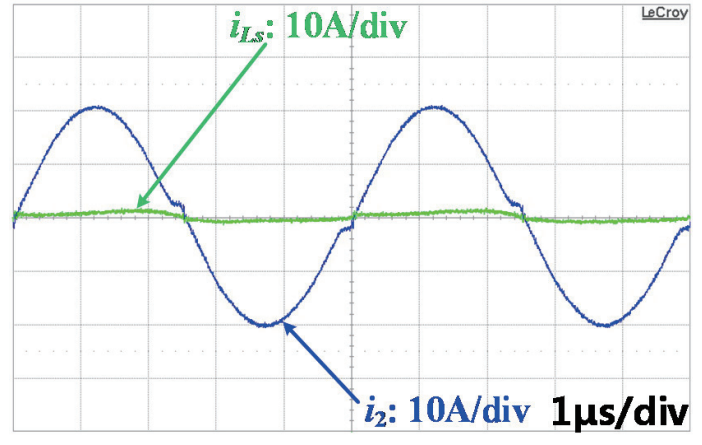

Fig. 21. Waveform of current in $L_{\mathrm{s}}$.

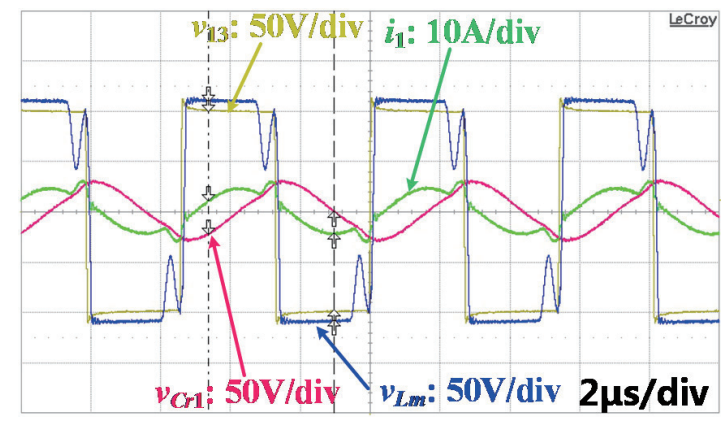

Fig. 22. Waveform under $1.2 \mathrm{~kW}$ (light load).

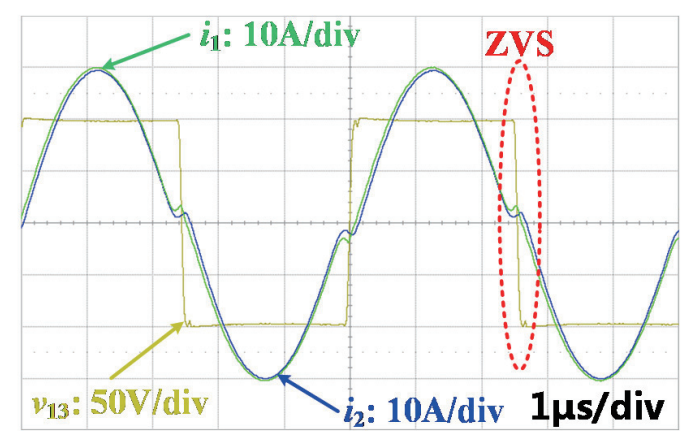

Fig. 23. Waveform under rated $3.6 \mathrm{~kW}$ (full load).

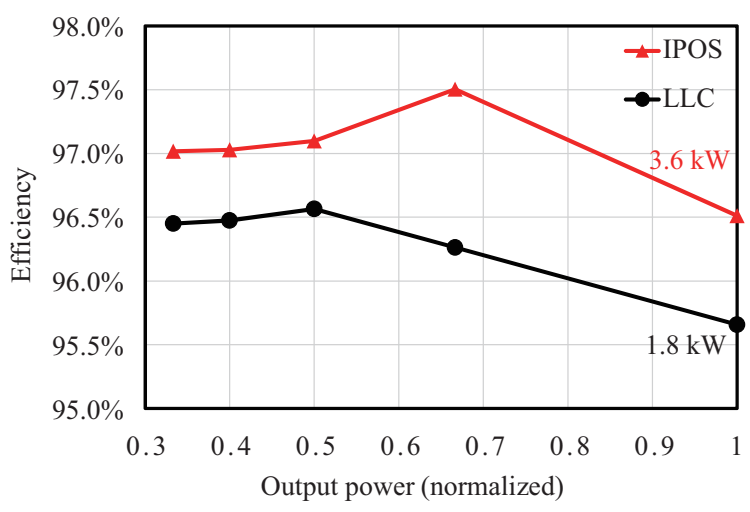

Fig. 24. Efficiency curves.

and $1.2 \mathrm{kV}$ output voltage, high gain and high efficiency are achieved for the IPOS LLC converter.

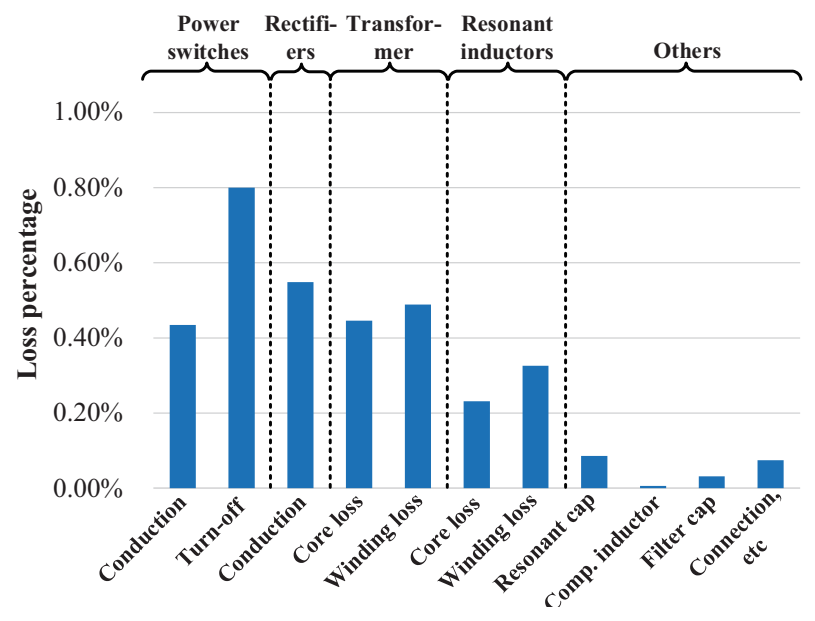

Fig. 25. Loss breakdown under $3.6 \mathrm{~kW}$ load.

Based on the experimental results, the loss breakdown of the prototype under $3.6 \mathrm{~kW}$ full-load condition is analyzed by theoretical calculation, as shown in Fig. 25. It is evident that the core loss of transformer is relatively small and the major loss is distributed at the conduction loss and turn-off loss of the power devices, which may be further improved by choosing devices with lower on-resistance and better performance on high frequency applications such as $\mathrm{SiC}$ and $\mathrm{GaN}$.

\section{CONCLUSION}

An IPOS LLC converter is proposed in this paper to deal with the high voltage gain and high efficiency applications. A high efficiency is obtained by utilizing a coupled transformer to implement the magnetic flux cancellation, and the size and power losses of the magnetic core are reduced and the output voltage is naturally balanced due to the common magnetic flux that flows through different windings. A bridge wire that interconnects resonant tanks is proposed to balance the current between modules. Furthermore, the current inside one module is balanced by the coordination of compensation inductor and delay control. The proposed structure has been verified on a $200 \mathrm{kHz} 100 \mathrm{~V} / 1.2 \mathrm{kV} 3.6 \mathrm{~kW}$ prototype. Experimental results show that the converter has a good performance on current sharing and can achieve high efficiency up to $97.5 \%$.

\section{REFERENCES}

[1] B. Dudley, "BP statistical review of world energy 2018," British Petroleum Co., London, U.K., 2018.

[2] Renewable Energy Policy Network for the 21st Century, "Renewables 2018 global status report," [Online]. Available: http://www.ren21.net/ publications.

[3] W. Chen, K. Zhuang, and X. Ruan, "A input-series- and output-parallelconnected inverter system for high-input-voltage applications," in IEEE Transactions on Power Electronics, vol. 24, no. 9, pp. 2127-2137, Sep. 2009.

[4] X. Hu and C. Gong, "A high gain input-parallel output-series DC/DC converter with dual coupled inductors," in IEEE Transactions on Power Electronics, vol. 30, no. 3, pp. 1306-1317, Mar. 2015.

[5] P. Wang, L. Zhou, Y. Zhang, J. Li, and M. Sumner, "Input-parallel output-series DC-DC boost converter with a wide input voltage range, 
for fuel cell vehicles," in IEEE Transactions on Vehicular Technology, vol. 66, no. 9, pp. 7771-7781, Sep. 2017.

[6] Jeong-il Kang, Chung-Wook Roh, Gun-Woo Moon, and Myung-Joong Youn, "Phase-shifted parallel-input/series-output dual converter for high-power step-up applications," in IEEE Transactions on Industrial Electronics, vol. 49, no. 3, pp. 649-652, Jun. 2002.

[7] J. Liu and K. W. E. Cheng, "New power sharing scheme with correlation control for input-parallel-output-series-based interleaved resonant inverters," in IET Power Electronics, vol. 7, no. 5, pp. 1266-1277, May 2014.

[8] X. Kong and A. M. Khambadkone, "Analysis and implementation of a high efficiency, interleaved current-fed full bridge converter for fuel cell system," in IEEE Transactions on Power Electronics, vol. 22, no. 2, pp. 543-550, Mar. 2007.

[9] A. J. Mason, D. J. Tschirhart, and P. K. Jain, "New ZVS phase shift modulated full-bridge converter topologies with adaptive energy storage for SOFC application," in IEEE Transactions on Power Electronics, vol. 23, no. 1, pp. 332-342, Jan. 2008.

[10] T. Mishima, K. Akamatsu, and M. Nakaoka, "A high frequency-link secondary-side phase-shifted full-range soft-switching PWM DC-DC converter with ZCS active rectifier for EV battery chargers," in IEEE Transactions on Power Electronics, vol. 28, no. 12, pp. 5758-5773, Dec. 2013.

[11] S. Yu, M. Q. Nguyen, and W. Choi, "A novel soft-switching battery charge/discharge converter with the zero voltage discharge function, " in IEEE Transactions on Power Electronics, vol. 31, no. 7, pp. 5067-5078, Jul. 2016.

[12] H. Wu, L. Chen, and Y. Xing, "Secondary-side phase-shift-controlled dual-transformer-based asymmetrical dual-bridge converter with wide voltage gain," in IEEE Transactions on Power Electronics, vol. 30, no. 10, pp. 5381-5392, Oct. 2015.

[13] S. Shao, H. Chen, X. Wu, J. Zhang, and K. Sheng, "Circulating current and ZVS-on of a dual active bridge DC-DC converter: a review," in IEEE Access, vol. 7, pp. 50561-50572, Apr. 2019.

[14] S. Shao, M. Jiang, W. Ye, Y. Li, J. Zhang, and K. Sheng, "Optimal phaseshift control to minimize reactive power for a dual active bridge DC-DC converter," in IEEE Transactions on Power Electronics, vol. 34, no. 10, pp. 10193-10205, Oct. 2019.

[15] B. Yang, F. C. Lee, A. J. Zhang, and G. Huang, "LLC resonant converter for front end DC/DC conversion," in Proceedings of APEC Seventeenth Annual IEEE Applied Power Electronics Conference and Exposition(Cat. No. 02 CH 37335), Dallas, TX, USA, 2002, pp. 11081112, vol. 2.

[16] B. Lu, W. Liu, Y. Liang, F. C. Lee, and J. D. Van Wyk, "Optimal design methodology for LLC resonant converter," in Proceedings of Twenty-First Annual IEEE Applied Power Electronics Conference and Exposition, 2006. APEC'06, Dallas, TX, 2006. pp. 6.

[17] W. Chen, K. Zhuang, and X. Ruan, "A input-series- and output-parallelconnected inverter system for high-input-voltage applications," in IEEE Transactions on Power Electronics, vol. 24, no. 9, pp. 2127-2137, Sep. 2009.

[18] H. Chen, X. Wu, and S. Shao, "A current-sharing method for interleaved high-frequency LLC converter with partial energy processing," in IEEE Transactions on Industrial Electronics, vol. 67, no. 2, pp. 1498-1507, Feb. 2020.

[19] H. Wang, Y. Chen, Yan-Fei Liu, and P. C. Sen, "A general multi-phase coupled-resonant-tank resonant converter," in Proceedings of 2018 IEEE Applied Power Electronics Conference and Exposition (APEC), San Antonio, TX, 2018, pp. 2183-2190.

[20] H. Wang, Y. Chen, B. Sheng, Yan-Fei Liu, and P. C. Sen, "Two-phase three-dimension common inductor LLC resonant converter with automatic current sharing," in Proceedings of 2018 IEEE Applied Power Electronics Conference and Exposition (APEC), San Antonio, TX, 2018, pp. 3575-3582.

[21] H. Wang, Y. Chen, Y. Qiu, P. Fang, Y. Zhang, L. Wang, Y. Liu, J. Afsharian, and Z. Yang, "Common capacitor multiphase LLC converter with passive current sharing ability," in IEEE Transactions on Power
Electronics, vol. 33, no. 1, pp. 370-387, Jan. 2018.

[22] D. Huang, S. Ji, and F. C. Lee, "LLC resonant converter with matrix transformer," in IEEE Transactions on Power Electronics, vol. 29, no. 8, pp. 4339-4347, Aug. 2014.

[23] C. Oeder and T. Duerbaum, "ZVS investigation of LLC converters based on FHA assumptions," in Proceedings of 2013 Twenty-Eighth Annual IEEE Applied Power Electronics Conference and Exposition (APEC), Long Beach, CA, 2013, pp. 2643-2648.

[24] J. Liu, J. Zhang, T. Q. Zheng, and J. Yang, "A modified gain model and the corresponding design method for an LLC resonant converter," in IEEE Transactions on Power Electronics, vol. 32, no. 9, pp. 6716-6727, Sep. 2017.

[25] Q. Wang, X. Zhang, R. Burgos, D. Boroyevich, A. White, and M. Kheraluwala, "Design considerations for a high efficiency $3 \mathrm{~kW}$ LLC resonant DC/DC transformer," in Proceedings of 2015 IEEE Energy Conversion Congress and Exposition (ECCE), Montreal, QC, 2015, pp. 5454-5461.

[26] B. Li, Q. Li, F. C. Lee, Z. Liu, and Y. Yang, "A high-efficiency highdensity wide-bandgap device-based bidirectional on-board charger," in IEEE Journal of Emerging and Selected Topics in Power Electronics, vol. 6, no. 3, pp. 1627-1636, Sep. 2018.

[27] C. Fei, F. C. Lee, and Q. Li, "High-efficiency high-power-density LLC converter with an integrated planar matrix transformer for high-output current applications," in IEEE Transactions on Industrial Electronics, vol. 64, no. 11, pp. 9072-9082, Nov. 2017.

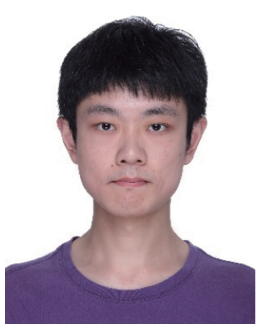

Yucen Li was born in Hubei, China, in 1995. He received the B.S. degree in Electrical Engineering from Zhejiang University, Hangzhou, China, in 2017, and is currently pursuing the M.S. degree at the College of Electrical Engineering, Zhejiang University. His research interest include with highefficiency DC/DC converters.

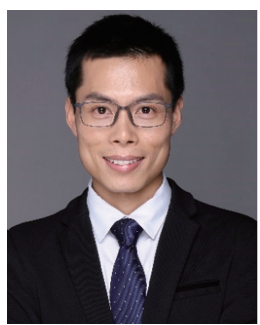

Shuai Shao received the B.S. degree from Zhejiang University, China, in 2010, and the Ph.D. degree in Electrical and Electronic Engineering from the University of Nottingham, U.K., in 2015.

In 2015, he joined the College of Electrical Engineering, Zhejiang University, as a Lecturer. His research interests include solid-state transformers, bidirectional dc-de converters, and fault detection in power converters.

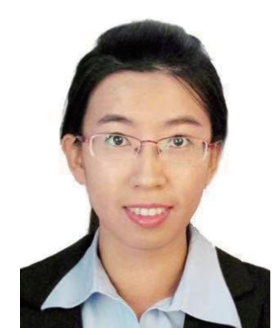

Hui Chen was born in Zhejiang, China, in 1988. She received the B.S. and Ph.D. degrees in Electrical Engineering from Zhejiang University, Hangzhou, China, in 2010 and 2015, respectively.

She is currently with the Zhejiang University City College, Hangzhou. Her current research interests include high-efficiency dc-dc converters and topology research. 


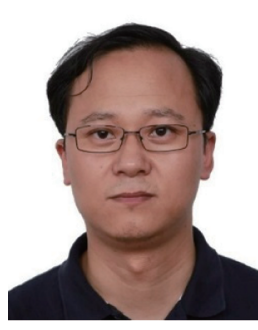

Junming Zhang received the B.S., M.S., and Ph.D. degrees from Zhejiang University, Hangzhou, China, in 1996, 2000 and 2004, respectively, all in Electrical Engineering.

He is currently a Professor at the College of Electrical Engineering, Zhejiang University. From 2010 to 2011, he was a Visiting Scholar in the Department of Electrical and Computer Engineering, Michigan State University, East Lansing. His research interests include power electronics system integrations, power management, and high-efficiency converters.

He is an Associate Editor of the IEEE Transactions on Industry Applications and CPSS Transactions Power Electronics and Applications.

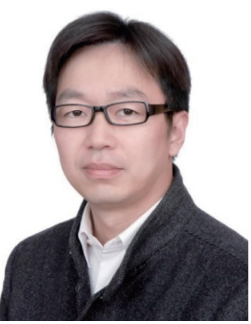

Kuang Sheng received the B.Sc. degree in Electrical Engineering from Zhejiang University, Hangzhou, China, in 1995, and the Ph.D. degree in Electrical Engineering from Heriot-Watt University, Edinburgh, U.K., in 1999.

He was a Postdoctoral Research Associate at Cambridge University, Cambridge, U.K., between 1999 and 2002. He was at Rutgers University, New Brunswick, NJ, USA, where he was an Assistant Professor and a tenured Associate Professor from 2002 to 2009. He is currently at Zhejiang University as a Chair Professor. He led a team that pioneered research works in $\mathrm{SiC}$ power ICs, $\mathrm{SiC}$ super-junction power devices and $\mathrm{GaN}$ vertical power devices. He has authored or coauthored over 200 technical papers in international journals and conferences and is a holder of 20 patents. His research interests include all aspects of power semiconductor devices and ICs on $\mathrm{SiC}, \mathrm{GaN}$ and $\mathrm{Si}$. Prof. Sheng serves as an Associate Editor of the IEEE Transactions on Power Electronics and the IEEE Transactions on Industry Applications. He served as the technical program chair of International Symposium on Power Semiconductor Devices and ICs (ISPSD) in 2015 and serves as the general chair for ISPSD in 2019. 